\title{
Early diagnosis of breast cancer - in respect to expectations of participants in screening programs and to contemporary research in imaging methods
}

Heino Hille

\begin{abstract}
On the background of new research results in screening of breast cancer, together with the expectation of the participants in a screening for breast cancer, the conventional mammography requires supplementation by means of tomosynthesis or additive ultrasound. Alternatively, ultrasound now seems to be an independent method of early detection of breast cancer because of its superior sensitivity, especially in the case of aggressive mammary carcinoma types. The MRI remains at present still a preferred method in high-risk cases and as an additive examination in case of insufficient presentation of the glandular tissue by conventional methods. MRI is also preoperatively valuable for more accurately measuring the extent of multifocal carcinomas.
\end{abstract}

\section{Key words}

brest cancer screening, mammography, breast ultrasound, breast MRI

\section{Aim}

This paper focuses on contemporary results of research with regard to the three main modalities in breast imaging: mammography, breast ultrasound and MRI.

Recommendations for diagnostic procedures are orientated on expectations of the participant in screening in relation to contemporary research.

\section{Introduction}

Although many women affected by life can be saved by diagnostics and therapy of breast cancer, one woman dies on this tumor every 30 minutes due to the frequency of the disease in Germany (statistically) [1].

This relationship leads to the need for further progress in diagnostics and therapy. We are looking at the progress made in early diagnosis for breast cancer by established imaging methods. 
The expectations of the woman participating in a screening examination on breast cancer may be as follows:

1. The tumor is to be discovered with high certainty in an early stage with good healing prospects.

2. Many "false alarms" (false positive findings) should not occur.

3. The method of examination should not be very unpleasant or painful.

4. The method of examination should not be inherently risk-prone.

These expectations cannot all be satisfied at the same time; it must be about getting as close as possible to it. Because, however, there is a gap between expectations and reality in the detection of breast cancer, therefore good information before the examination is important for the participating woman.

The main feature of the early detection method from the participant's point of view is its sensitivity, i.e. how accurate is it in its ability to detect tumors early not overlooking small carcinomas. Special attention is also given to sensitivity since laboratory diagnostics are at risk of taking early diagnosis in their competence.

Sensitivity is weighted against specificity, i.e. the property that negative classified findings are actually negative. In general, an inverse ratio of these parameters is to be expected in medical diagnostics: If the sensitivity is increased, the specificity decreases and vice versa.

Before introducing a diagnostic method, an acceptable ratio of sensitivity and specificity - expressed by the positive predictive value (PPV) - is mandatory.

From an epidemiological and economic perspective, a good PPV is the most important parameter for health policymakers and health care providers prior to the introduction of a diagnostic test into general health care.

For the individual patient, who takes part of a screening examination, the sensitivity of the method - an actual disease is not overlooked - might be in the forefront. Secondly, she does not want to be unnecessarily worried

\section{Advances in imaging methods}

According to the X-ray regulation - in Germany -, screening mammography is strictly permitted only at an age of 50 and can only be carried out in the recognized screening centers. Issuing curative referrals or conducting the examination as individual health care circumvents these legal requirements. If one looks at the performance of 
mammography, its sensitivity in women with breast density 1 (A) and 2 (B) (fat-rich mammals) is good to very good; its specificity is also satisfying.

However, the sensitivity of mammography is steeply lower the more densely the individual breast is built up and decreases in women with high density to $50 \%$ or less [2, 3]. Since a high proportion of women, $50 \%$ and more have a breast density above 2 (B) $[4,5]$; therefore mammography is not general, but only sufficient for women with density 1 (A) and 2 (B).

Especially voluminous breasts with a small gland part benefit from mammography because of its good overview. Painful sensations caused by the pressing and the properties of the ionizing radiation are from the point of view of the women disadvantages of the method.

In order to compensate for the low sensitivity of mammography in the dense mammary, a new technique has been developed with 3D mammography - tomosynthesis. With tomosynthesis, the mammary is scanned radiographically in individual thin layers (under $1 \mathrm{~mm}$ ) from different angles, so that the problem of superimposition of the structures in conventional mammography is solved (Fig. 1,2).

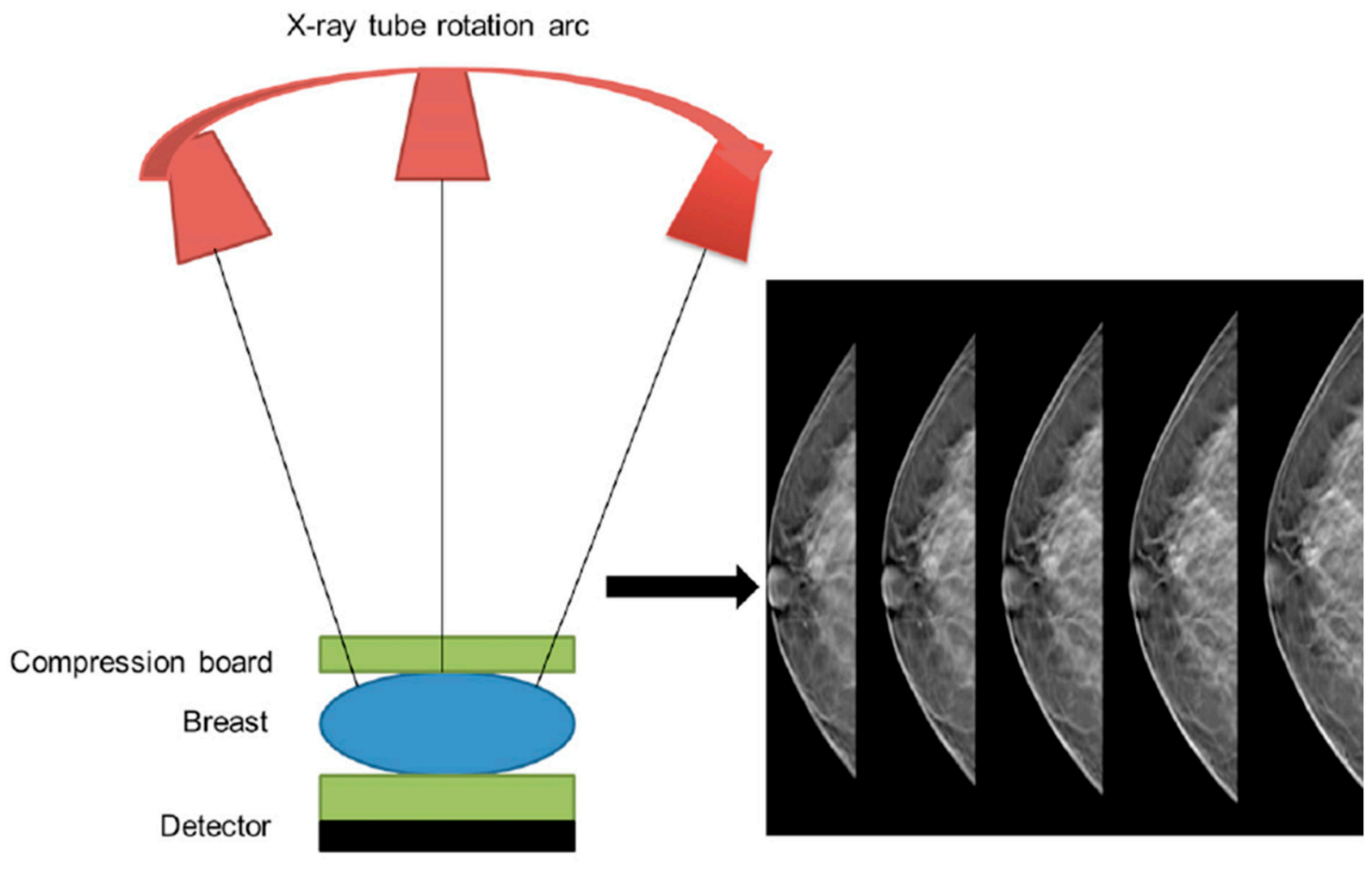

(a)

(b)

Figure 1: Tomosynthesis scans the breast from different angels creating thin layers without superimposition (Figure Hologic Inc.; with permission) 


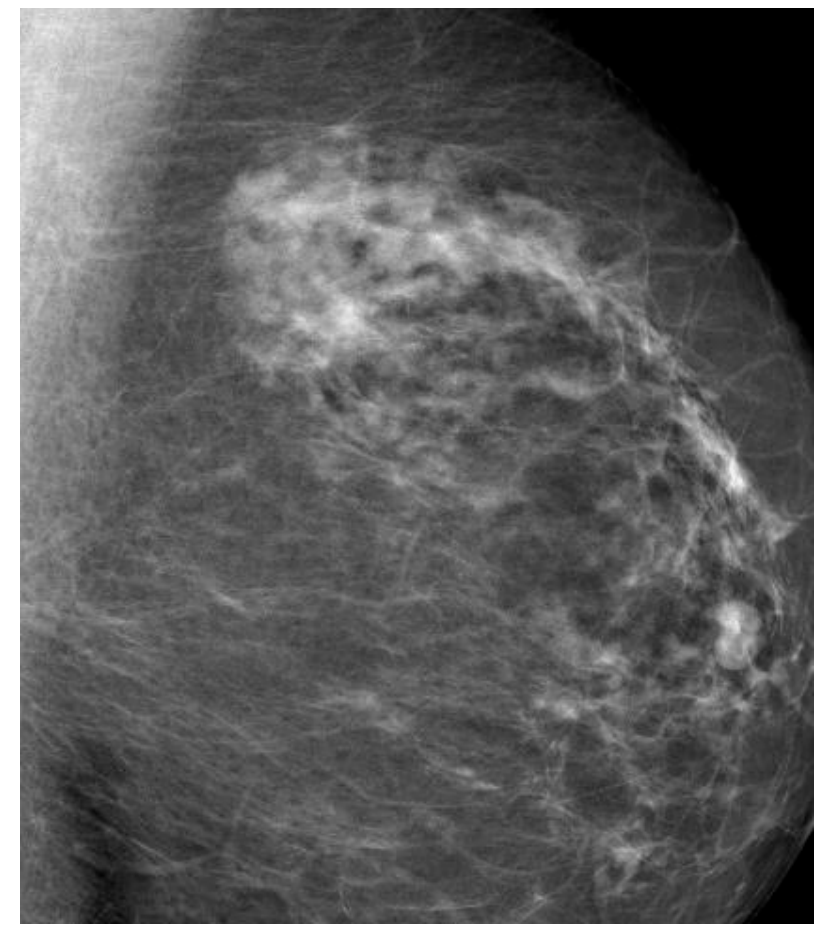

a

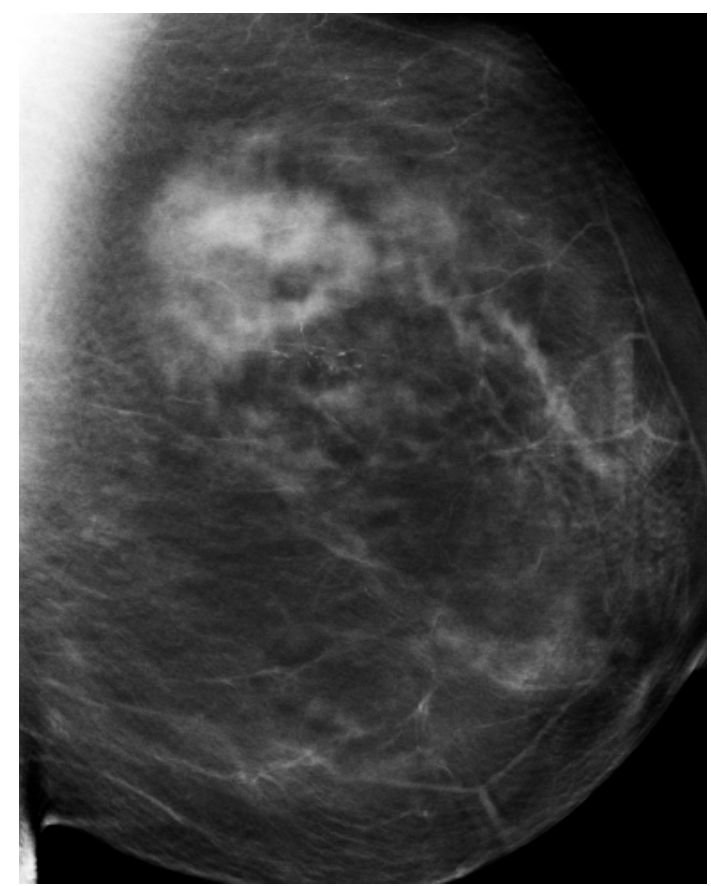

C

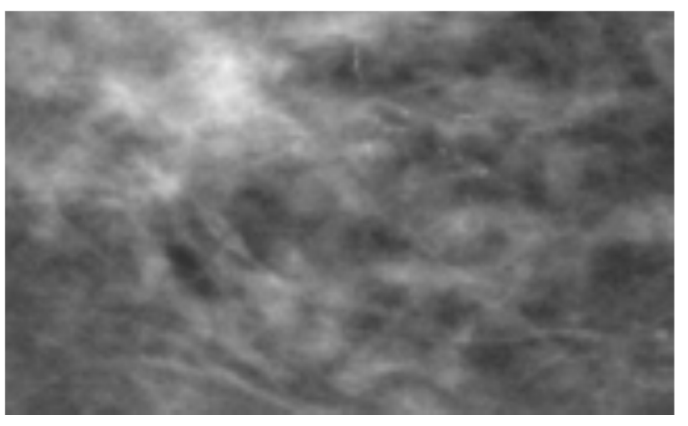

b

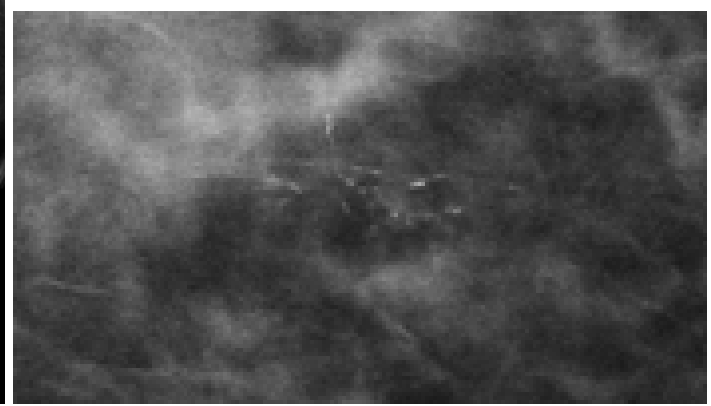

d

Figure 2, a-d

a: 2D mammography , b: lupe zoom (small picture), microcalcifications not visible; c: tomosynthesis layer ,same patient, d: clearly microcalcifications (lupe zoom). (Figures Dr. Rückner, Mammadiagnostik, Hamburg, with permission) 
Whether tomosynthesis should be carried out without the conventional mammography or only in adjunct in the case of dense breasts is still a subject of discussion in radiology [6]. The radiation dose is different for the devices used - in some cases equal to a 2-plain mammogram, in others higher - any additional use of tomosynthesis must also take an additional radiation exposure into account.

Tomosynthesis can increase the sensitivity of 2-plain- mammography by up to $40 \%$ in dense breasts $[7,8]$. A particular positive feature appears to be its accompanying increase in specificity, so that the use of tomosynthesis may generate less unnecessary clarification cases. The main gain in diagnostic capacity is in the collective of women with breast density $3(\mathrm{C})$, whereas the sensitivity at very high density also decreases in the case of tomosynthesis [9-11].

Ultrasound has the advantage that it is not dependent on the density of the breast in its performance and there are no restrictions on the age of the patient. Furthermore, it can be carried out in a decentralized and cost-effective manner and also in gynecological practices. It is becoming increasingly apparent that its sensitivity is superior in the normal collective, but especially in the clientele with dense breasts. The sensitivity of ultrasound in recent comparative publications is about $20 \%$ higher than that of mammography, can be up to $40 \%$ in defined collectives [12-19].

Disadvantage is its subjectivity (observer dependency) and its lower specificity (higher number of false positive findings).

The use of automated volume scanners (ABUS) could now be used to reduce subjectivity.

In respect to inferior specificity, it must be taken into account that BIRADS 3 (very probably benign) findings (mainly fibroadenomas) are usually counted to be false positive, which is questionable. If BIRADS 3 findings are counted as correct negative, there must be no significant difference in the specificity of ultrasound compared to mammography $[17,20]$.

Magnetic resonance imaging (MRI) is the most accurate method in its sensitivity and is superior to the other methods, particularly in women with very complex architecture of the breasts as well as in high-risk collectives.

According to the latest findings, it may also be appropriate in the normal collective to provide better early detection than all other methods together, and possibly even at greater intervals (3 years) [21]. MRI becomes increasingly important preoperative to 
determine the exact extent, especially of multifocal carcinomas. Both mammography and ultrasound often underestimate intraductal spread, not only in lobular carcinomas. MRI, however, is only available to a limited extent in a high degree of expertise, is significantly more expensive and requires a (invasive) contrast injection. Concerns about adverse effects of the contrast medium for the brain and kidneys are not eliminated [22].

Approximately equivalent results to MRT can be achieved with contrast-enhanced mammography (CESM) [23], but until now, this method is only available in a few places.

The sensitivity of the method in general is not the only important aspect, but also - with a view to reducing the risk of breast cancer mortality - its competence to diagnose the aggressive types of breast cancer is at the forefront. The large older screening trials had not yet been able to make any distinctions. The Luminal A, Luminal B, Her 2 neu positive, Triple negative, more precise: basal cell type- we now have criteria to distinguish tumor types from their malignant potential. If the diagnostic competence is correlated with these types of tumors, we have further criteria that assign methods to their relevance to mortality reduction. According to the present studies, ultrasound is superior in the diagnosis of basal cell carcinomas due to the more frequent absence of typical mammographic signs of malignancy [24-47].

\section{Current recommendations on the background of new developments}

If mammography is performed as a screening examination, tomosynthesis should be available for women with a higher density of the breast or an ultrasound examination (possibly with automatic whole breast volume scanning - ABUS) should be connected in adjunct.

If the woman participates in organized mammographic screening with a two-year interval, she should take an ultrasound examination in the year between - for reasons of tumor growth: a two-year examination interval is too long for aggressive mammary carcinomas. If an individual gland density 4 (D) is present, an additional ultrasound examination should be performed annually.

Ultrasound additive to mammography detects more additional carcinomas than additive tomosynthesis. Ultrasound achieves better results in cases with extreme density and with carcinomas with conventional as benign recognized features [9-11]. 
Nowadays, as an early detection method for all age groups, irrespective of the density of the gland, qualified ultrasound as its own is an available and cost-effective method.

Whether this is carried out in the form of the classical handheld ultrasound (HHUS) or in the form of the automatic volume scanner (ABUS) is probably not essential $[48,49]$. Both methods have advantages and disadvantages. Additional modules such as color Doppler and elastography, as well as the special dynamics of the HHUS, especially in the examination of lesion findings are advantages of the HHUS. ABUS is less dependent on the examiner and the ability to scan through the entire breast in the supine plane (Cplane) may reduce the risk of false negatives.

The quality and experience of the investigator determines the percentage in which a complementary method is recommended. For BIRADS 0 cases, e.g. in the case of very inhomogeneous and/or sound-absorbing parenchyma, the recommendation for MRI would be given. In the case of a localized structural abnormality with irregular mammary gland architecture or a total inhomogeneous sonographic view, mammography should be used to exclude a DCIS.

Ultrasound has a weakness with respect to some forms of DCIS, as it does not recognize finely distributed microcalcifications without hypoechoic environmental structures. On the other hand, ultrasound detects mass-like DCIS (with and without microcalcifications) better than mammography and can also recognize forms with gross calcifications $[50,51,52]$. It is advisable to draw the patient's attention to this possible gap in ultrasound diagnostics and to suggest mammography in addition if early diagnosis of DCIS is the target.

Of course one will get the best results if all methods are used in a complementary way. But neither from the diagnostic necessity, nor from an economic point of view, does this appear justified in general.

For extremely complex mammals or high-risk patients, an MRI should be carried out at regular intervals (1-2 years).

If ultrasound, as described above, is a screening method suitable for all women $[13,17]$, why breast ultrasound is recommended mainly as a supplementary examination (complementary to mammography)?

The following three reasons are listed regularly:

1. Only mammography has demonstrated an effect on mortality in breast cancer in large randomized studies. 
2. The use of ultrasound leads to a lower positive predictive value (PPV) due to an increase in false positive results with subsequent further investigation procedures, which also leads to cost increases.

3. A quality assurance of ultrasound application was not given.

To 1: The large randomized mammographic screening studies with a follow-up period of 10 or more years, which were carried out in the second half of the 20th century, are not likely to be reissued with other modern methods. The argument refers to a methodological standard over 30 years old and cemented it. But science has, by its very nature, a progressive-dynamic character. Today, the methods are to compare to how well they diagnose breast carcinomas in an early stage and which type of tumors they recognize well or less well.

To 2: As stated above, the specificity of ultrasound does not have to differ significantly in mammography if the probably benign findings (BIRADS 3) are counted as being negative.

The ultrasound examination is offered much cheaper than mammography. An additional complementary examination is much less frequently required in primary ultrasound diagnostics with good quality than in primary mammography because of high density. In addition, the gain in life-years must be taken into account by the carcinomas diagnosed only by ultrasound (Fig. 3). 


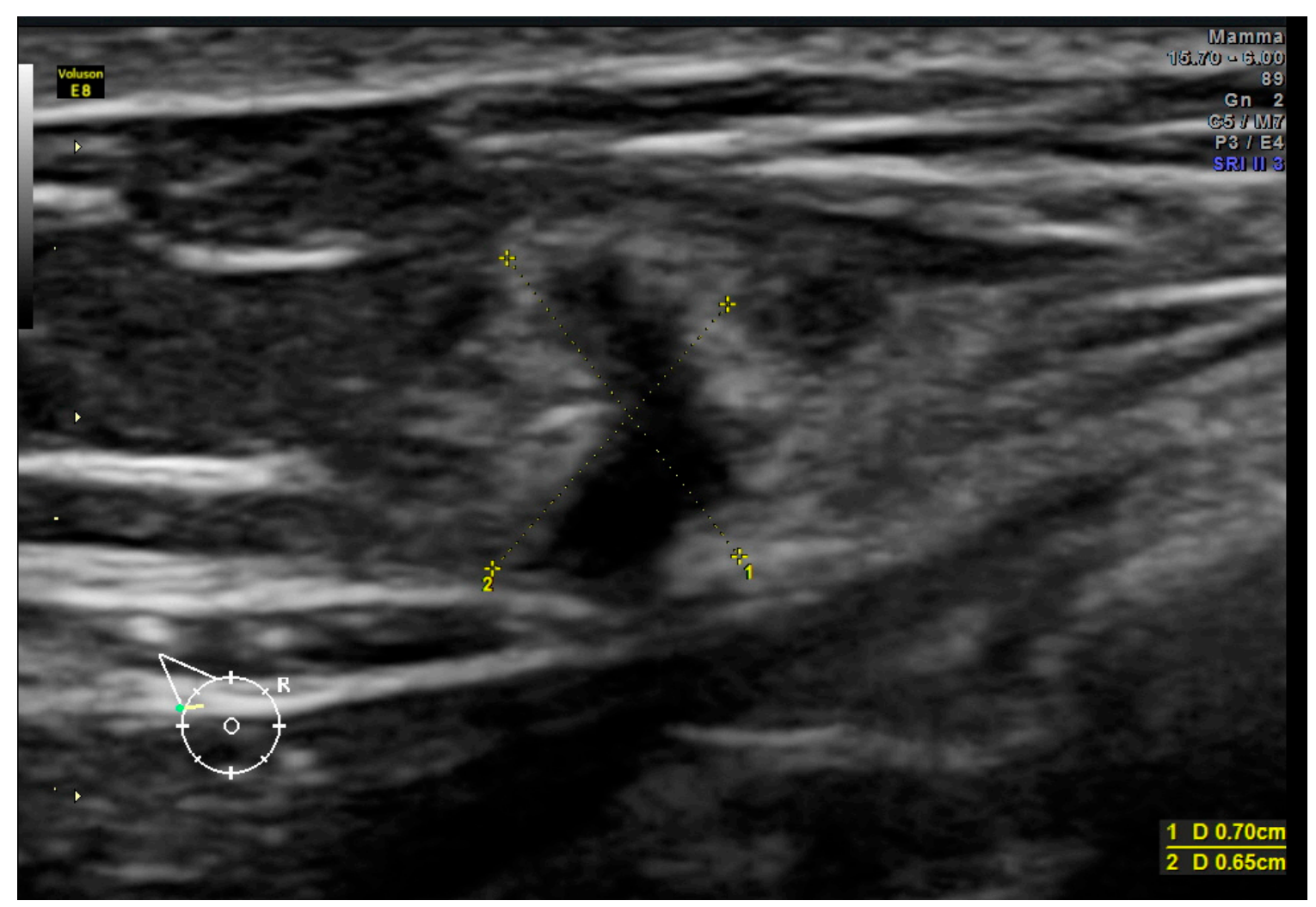

Figure 3:

50-year-old patient with an induration located upper outer site right breast. Clearly visible lesion by ultrasound, BIRADS V; Mammography: no suspicious findings, BIRADS II; MRI: small nodular contrast enhancements, not suspect. Ultrasound-guided core biopsy: invasive ductal carcinoma (NST)

It is certain that more biopsies are induced by sonography, since BIRADS 3 findings are often invasively clarified (at the request of the patient).

To 3: Expertise levels can also be established in breast ultrasound by means of performance assessment. In general, in the case of sophisticated methods - diagnostic or therapeutic - good results can only be achieved with good quality, in this respect ultrasound is not a special feature.

The fact that decentralized breast ultrasound in offices can be performed sufficiently is well documented $[53,54]$.

In summary: Mammographic screening needs a supplement or replacement by tomosynthesis or an ultrasonic examination in adjunct in the case of dense breast. 
Alternatively, ultrasound nowadays seems to be an independent imaging method irrespective of age and density. To establish ultrasound as a method of early detection of breast carcinoma by its own, larger trials with ultrasound application with means of new technologies and mammography in adjunct should be initiated to find out to which extent ultrasonography alone may miss small carcinomas and if mammography remains necessary together with breast ultrasound. MRI is likely to play a greater role in early detection of breast cancer in the future.

The author declares no conflict of interest.

References

1. Zeichen aus dem Blut, Zeit 2015; 35 (German)

2. Winkler NS, Raza S et al. Breast density: clinical implications and assessment methods. RadioGraphics 2015; 35:316-324

3. Checka CM, Chun JE et al. The relationship of mammographic density and age: implications for breast cancer screening. AJR 2012; 198:292-295

4. Kolb TM, Lichy J et al. Comparison of the performance of screening mammography, physical examination, and breast US and evaluation of factors that influence them: an analysis of 27,825 patient evaluations. Radiology 2002; 225:165-175

5. Pisano ED, Gatsonis C et al. Diagnostic performance of digital versus film mammography for breast-cancer screening. N Engl J Med 2005; 353: 1773-1783

6. Pisano ED, Yaffe MJ. Breast cancer screening. Should tomosynthesis replace digital mammography? JAMA 2014; 311:2488-2489

7. Skaane P. Breast cancer screening with digital breast tomosynthesis. Breast Cancer 2017; 24: 32-41

8. Houssami N, Turner RM. Rapid review: Estimates of incremental breast cancer detection from tomosynthesis (3D-mammography) screening in women with dense breasts. Breast 2016; 30:141-145

9. Nam KJ, Han BK et al. Comparison of full-field digital mammography and digital breast tomosynthesis in ultrasonography-detected breast cancers. Breast 2015; 24:649-655

10. Kim WH, Chang JM et al. Diagnostic performance of tomosynthesis and breast ultrasonography in women with dense breasts: a prospective comparison study. Breast Cancer Res Treat 2017; 162: 85-94 
11. Tagliafico AS, Clabrese M et al. Adjunct screening with tomosynthesis or ultrasound in women with mammography-negative dense breasts: interim report of a prospective comparative trial. J Clin Oncol 2016; 345: 1882-1888

12. Tamaki $\mathrm{K}$, Ishida $\mathrm{T}$ et al. Breast ultrasonographic and histopathological characteristics without any mammographic abnormalities. Jpn J Clin Oncol 2012; 242: $168-174$

13. Cho KR, Seo BK et al. Breast cancer detection in a screening population: comparison of digital mammography, computer-aided detection applied to digital mammography and breast ultrasound. J Breast Cancer 2016; 19: 316-323

14. Hwang JY, Han BK et al. Screening ultrasound in women with negative mammography: outcome analysis. Yonsei Med J 2015; 56: 1352-1358

15. Tan KP, Azlan ZM et al. The comparative accuracy of ultrasound and mammography in the detection of breast cancer. Med J Malaysia 2014; 69: 79-85

16. Ohuchi, N, Suzuki A et al. Sensitivity and specificity of mammography and adjunctive ultrasonography to screen for breast cancer in the Japan strategic anti-cancer randomized trial (J-Start): a randomized controlled trial. Lancet 2015, http://dx.doi.org/10.1016/S0140-6736(15)00774-6

17. Shen $\mathrm{S}$, Zhou Y et al. A multi-center randomized trial comparing ultrasound vs. mammography for screening breast cancer in high-risk Chinese women. BJC 2015; 112:998-1004

18. Wilczek B, Wilczek HE et al. Adding 3D automated breast ultrasound to mammography screening in women with heterogeneously and extremely dense breasts: report from a hospital based, high-volume, single-center breast cancer screening program. Eur J Radiol 2016; 85:1554-1563

19. Weigert JM. The Connecticut experiment; the third installment: 4 years of screening women with dense breasts with bilateral ultrasound. Breast J 2017; 23: 34-39

20. Hille $\mathrm{H}$, Vetter M et al. The accuracy of BI-RADS classification of breast ultrasound as a first-line imaging method. Ultraschall in Med 2012; 33:160-163

21. Kuhl CK, Strobel K et al. Supplemental breast MR imaging screening of women with average risk of breast cancer. Radiology 2017; 283:361-370 doi:10.1148/radiol.2016161444

22. Kandar T, Nakai Y et al. Gardolinium deposition in the brain, Magn Reson Imaging 2016; 34:1346-1350

23. Fallenberg EM, Schmitzberger FF et al. Contrast-enhanced spectral mammography vs. mammography and MRI - clinical performance in a multireader evaluation. Eur Radiol 2016; doi:10.1007/s00330-016-4650-6

24. Prat A, Prineda E.et al. Clinical implications of the intrinsic molecular subtypes of breast cancer. The Breast 2015; S6-S35

25. Lin CY, Barry-Holston KQ et al. Breast cancer stem cells: are we ready to go from bench to bedside? Histopathology 2016; 68: 119-137

26. Jung HA , Park YH et al. Prognostic relevance of biological subtype overrides that of TNM staging in breast cancer: discordance between stage and biology. Tumor Biol 2015; 36: 1073-1079 
27. Pan B, Yao R et al. Clinicopathological characteristics and long-term prognosis of screening detected non-palpable breast cancer by ultrasound in hospital-based Chinese population (2001-2014). Oncotarget 2016; 7: 38864-38875 Doi: 10. 18632/oncotarget.12319

28. Tsau HS, Yen AM et al. Mammographic tumour appearance and triple-negative breast cancer associated with long-term prognosis of breast cancer death: a Swedish cohort study. Cancer Epidemiol. 2015; 39: 200-208

29. Kim SY, Seo BK et al. Correlation of ultrasound findings with histology, tumor grade, and biological markers in breast cancer. Acta Oncol 2008; 47:1531-1538

30. Ko E, Lee AH et al. Triple-negative breast cancer: correlation between imaging and pathological findings. EurRadiol 2010; 20: 1111-1117

31. Choi YJ, Seong MH et al. Ultrasound and clinicopathological characteristics of triple-receptor-negative breast cancers. J Breast Cancer 2011; 14:119-123

32. Irshad A, Leddy $\mathrm{R}$ et al. Assessing the role of ultrasound in predicting the biological behavior of breast cancer. AJR 2013; 200: 284-290

33. Wojcinski S, Sollman AA et al. Sonographic features of triple-negative and nontriple-negative breast cancer. JUM 2012; 31: 1531-1541

34. Dogan BE, Turnbull LW et al. Imaging of triple-negative breast cancer. Ann Oncol 2012; 23 (Supplement 6): vi23-vi29

35. Kim MY, Choi N. Mammographic and ultrasonographic features of triple-negative breast cancer: a comparison with other breast cancer subtypes. Acta Radiol 54; 2013; 889-894

36. Aho M, Irshad A et al. Correlation of sonographic features of invasive ductal mammary carcinoma with age, tumor grade, and hormone-receptor status. J Clin Ultrasound 2013; 41: 10-17

37. Boissery-Lacroix M, MacGrogan G et al. Triple-negative breast cancers: associations between imaging and pathological findings for triple-negative tumors compared with hormone-receptor-positive/human epidermal growth factor receptor-2-negative breast cancers. The Oncologist 2013; 18: 802-811

38. Boissery-Lacroix M, Bullier B et al. Correlation between imaging and prognostic factors: molecular classification of breast cancers. Diagnostic and Interventional Imaging 2014; 95: 227-233

39. Li B, Zhao X et al. Associations between mammography and ultrasound imaging features and molecular characteristics of triple-negative breast cancer. Asian Pac J Cancer Prev 2014; 15:3555-3559

40. Gao B, Zhang H et al. Mammographic and clinicopathological features of triplenegative breast cancer, Br J Radiol 2014; 87: 20130496

41. Li Z, Ren M et al. The differences in ultrasound and clinicopathological features between basal-like and normal-like subtypes of triple negative breast cancer. PLoS ONE 2015; 10: e0114820. doi:10,1371/journal.pone.0114820

42. Yang Q, Liu HY et al. Ultrasonographic features of triple-negative breast cancer: a comparison with other breast cancer subtypes. Asian Pac J Cancer Prev 2015; 16: 3229-3232 
43. Çelebi F, Pilanci KN et al. The role of ultrasonographic findings to predict molecular subtype, histologic grade, and hormone receptor status of breast cancer. Diagn Interv Radiol 2015; 21:448-453

44. Jung HK, Han K et al. Mammographic and sonographic features of triple-negative invasive carcinoma of no special type. Ultrasound in Med \& Biol 2015; 41: 375383

45. Costantini M, Belli P et al. Association between sonographic appearances of breast cancers and their histopathologic features and biomarkers. J Clin Ultrasound 2015; 44: 26-33

46. Du HY, Lin BR et al. Ultrasonographic findings of triple-negative breast cancer. Int J Clin Exp Med 2015; 8:10040-10043

47. Li Z, Tian J et al. Differences in multi-modal ultrasound imaging between triplenegative and non triple-negative breast cancer. Ultrasound in Med \& Biol 2016; 42: 882-890. http://dx.doi.org/10.1016/j.ultrasmedbio.20125.12003

48. Wang HY, Jiang YX et al. Differentiation of benign and malignant breast lesions: a comparison between automatically generated breast volume scans and handheld ultrasound examinations. Eur J Radiol 2012:81:3190-3200

49. Kuzmiak CM, Ko EY et al. Whole breast ultrasound: Comparison oft the visibility of suspicious lesions with automated breast volumetric scanning versus handheld breast ultrasound. Acad Radiol 2015; 22:870-879

50. Chiang CL, Liang HL et al. Easily recognizable sonographic patterns of ductal carcinoma in situ oft the breast. Journal oft the Chinese Medical Association 2016; 79:493-499

51. Lee J, Lee JH et al. Non-mass lesions on screening breast ultrasound. Med Ultrason 2016; 18:446-451

52. Su X, Lin Q et al. Non-calcified ductal carcinoma in situ of the breast: comparison of diagnostic accuracy of digital tomosynthesis, digital mammography, and ultrasonography. Breast Cancer 2016; https://dx.doi.org/10.1007/s12282-016$\underline{0739-7}$

53. Lenz S. Breast ultrasound in office gynecology - ten years of experience. Ultraschall in Med 2011; https://dx.doi.org/10.1055/s-0029-1245426

54. Madjar H, Becker S et al. [Impact of breast ultrasound screening in gynecological practice]. Ultraschall in Med 2010; https://dx.doi.org/10.1055/s-0028-1110010 